\title{
Extragastrointestinal Stromal Tumor Mimicking Gastric Subepithelial Tumor
}

\author{
Se Won Kim, M.D. \\ Department of Surgery, Yeungnam University College of Medicine, Daege, Korea
}

Gastrointestinal stromal tumor (GIST) is the most common mesenchymal tumor of the gastrointestinal tract; however, it also occurs at a lower frequency in extra-gastrointestinal regions, such as omentum, mesentery, retroperitoneum, and undefined abdominal sites. This tumor is called an extragastrointestinal stromal tumor (EGIST). A 53-year-old woman presented with a gastric subepithelial tumor, which was incidentally detected by gastrofiberscopy. Contrast abdominal computed tomography (CT) showed a well-defined exophytic tumor with a minimally delayed peripheral enhancing nature near the gastrohepatic ligament. Surgical intervention was in- dicated, and laparoscopic surgery was planned. During surgery, no specific abnormality was found in the gastric wall and there was no evidence of a gastric subepithelial tumor. Based on immunohistochemical results, sensitive and specific immunostaining was observed for c-KIT, DOG- 1 , and PKC- $\theta$. In this case, the tumor was positive for CD-117(c-kit) and for smooth muscle actin expression.

Key words: Extragastrointestinal stromal tumor, CD 117, Gastric subepithelial tumor

\section{INTRODUCTION}

Gastrointestinal Stromal Tumors (GISTs) are a subset of GI mesenchymal tumors exhibiting various levels of differentiation. Previously, these tumors were classified as GI leiomyomas, leiomyosarcomas, leioblastomas, and schwannomas based on their histological findings and apparent origin in the muscularis propria layer of the intestinal wall. However, because of their immunohistochemical and ultrastructural characteristics, GISTs are now considered a distinct group of mesenchymal tumors. GISTs express c-kit protein, which is also known as CD117, which is considered a highly specific marker that differentiates GISTs from other mesenchymal tumors, such as, leiomyomas. ${ }^{1}$ Extragastrointestinal Stromal Tumors (EGISTs) are a rarely reported group of tumors that arise outside the GI but histologically resemble their GI counterparts. Approximately $80 \%$ are located in the omentum or mesentery, whereas the remaining $20 \%$ develop in the retroperitoneum. ${ }^{2,3}$ To our best knowledge, there are no definitive reports on EGIST arising from crus muscle. We present a rare case of EGIST mimicking

Received March 26, 2014, Revised August 7, 2014,

Accepted August 19, 2014

※ Corresponding author: Se Won Kim

Department of Surgery, Yeungnam University College of Medicine,

Yeungnam University Medical Center, Daemyeong 5-dong, Nam-gu,

Daege 705-717, Korea

Tel : +82-53-620-3580, Fax : +82-53-624-1213

E-mail : kswgs@ynu.ac.kr a exophytic gastric subepitelial tumor developed in the right crus muscle.

\section{CASE REPORT}

A 53-year-old woman presented with a gastric subepitelial tumor incidentally detected by gastrofiberscopy. Otherwise, the patient was in good health, with no unexplained fever, general ache, or weight loss. Physical examination findings were unremarkable. The initial biochemical and hematologic investigation, including complete blood count, electrolytes, liver function tests, and chest radiographic findings were all within normal ranges. Gastroscopic examination showed bulging mucosa, about $4 \times 3 \mathrm{~cm}$ in diameter in the posterior wall of the cardia (Fig. 1A). Endoscopic ultrasound of the stomach showed a $4.0 \times 3.0 \mathrm{~cm}$ sized hypoechoic lesion in the muscularis propria layer at the posterior wall of the cardia (Fig. 1B). Contrast abdominal computed tomography (CT) depicted a well-defined exophytic tumor with a minimally delayed peripheral enhancing nature near the gastrohepatic ligament (Fig. 2). These results lead us to consider a gastric subepitelial tumor, such as, GIST or a neurogenic tumor.

Surgical intervention was indicated, and laparoscopic surgery was promptly performed. During surgery no specific abnormality of the gastric wall or evidence of gastric subepitelial tumor was found. The tumor was found between the right crus muscle and the lesser curvature of the gastroesophageal junction. It was carefully dissected and the tumor of was easily separated from 

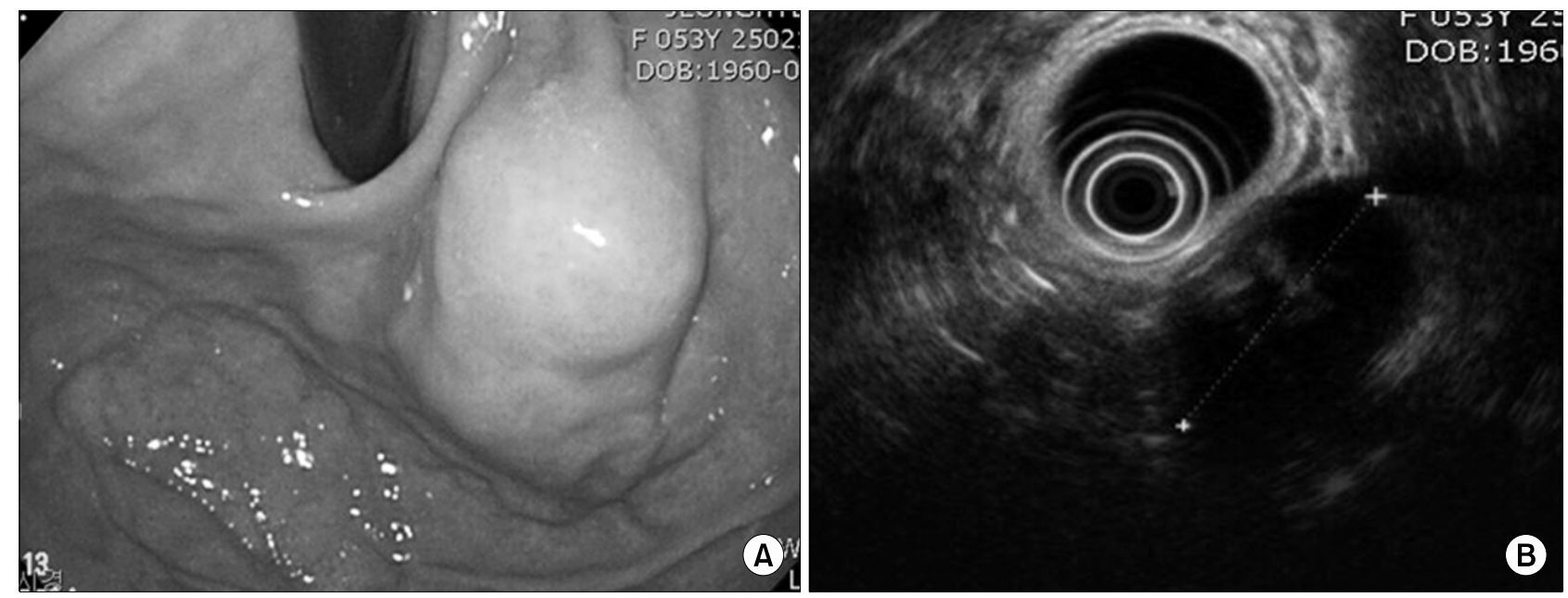

Fig. 1. Gastroscopic examination showed bulging mucosa, about $4 \times 3 \mathrm{~cm}$ in diameter in the posterior wall of the cardia (A) and endoscopic ultrasound of the stomach showed a hypoechoic lesion in the muscularis propria layer at the posterior wall of the cardia (B).
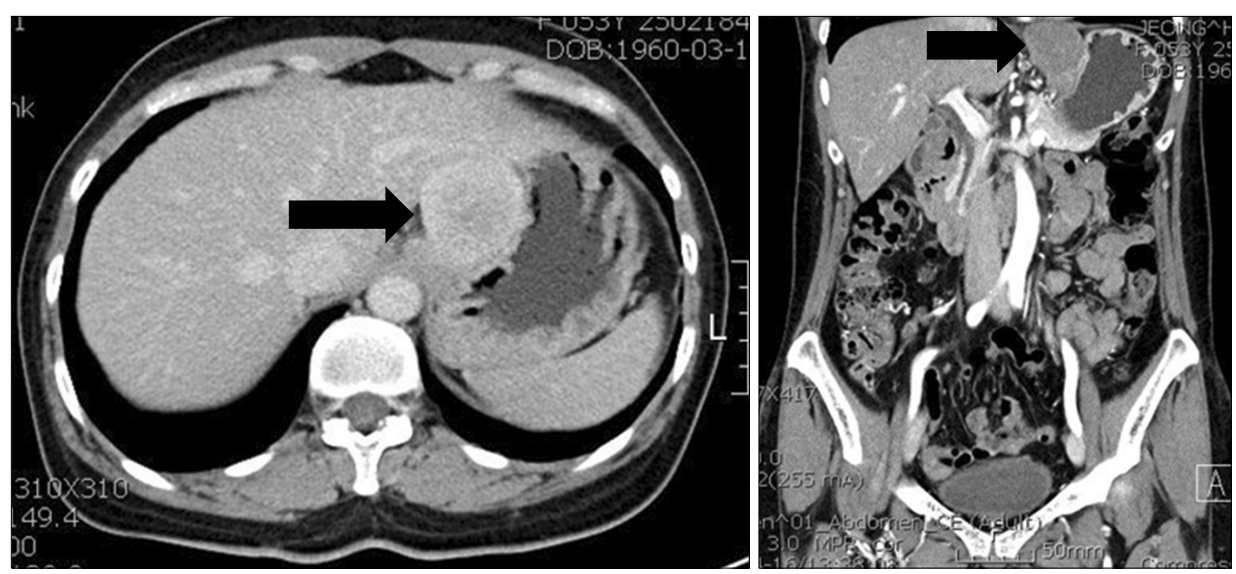

Fig. 2. Contrast abdominal computed tomography (CT) depicted a well-defined exogastric tumor with a minimally delayed peripheral enhancing nature near the gastrohepatic ligament (arrow).

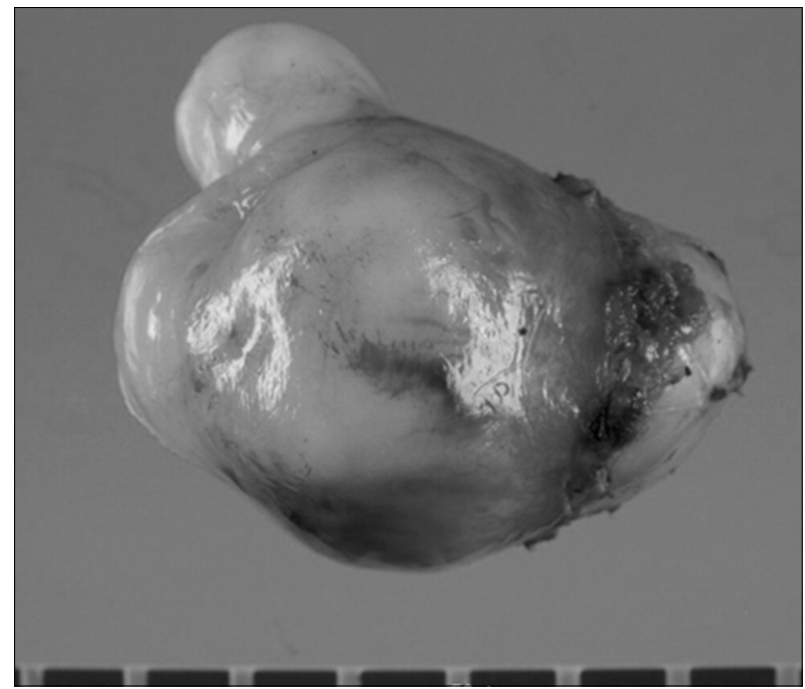

Fig. 3. The tumor specimen measured $5.2 \times 4.7 \times 3.8 \mathrm{~cm}$ and weighed $39.6 \mathrm{~g}$. the wall of stomach. There was no evidence of stomach wall origin tumor. It was also easily separated from the right crus muscle. So we dissected and removed it easily used to ultrasonic device. It was removed without event, and found to originate from the right crus muscle rather than the stomach wall. Frozen section examination showed it suspicious to be a gastrointestinal stromal tumor. So we carefully checked the dissected area but there was no remnant tumor and we finished the operation. The tumor specimen measured $5.2 \times 4.7 \times 3.8 \mathrm{~cm}$ and weighed $39.6 \mathrm{~g}$ (Fig. 3). The pathologic report was moderate risk gastrointestinal stromal tumor with less than 5/50 high power field (HPF), spindle cell type, no tumor necrosis, no tumor hemorrhage and smooth muscle differentiation. The immunohistochemistry features were weakly positive for CD-117 (c-kit) (Fig. 4A) and smooth muscle actin expression (Fig. 4B) but CD 34, desmin, S-100 protein and DOG-1 were negative. The patient's postoperative course was uneventful, and she con- 

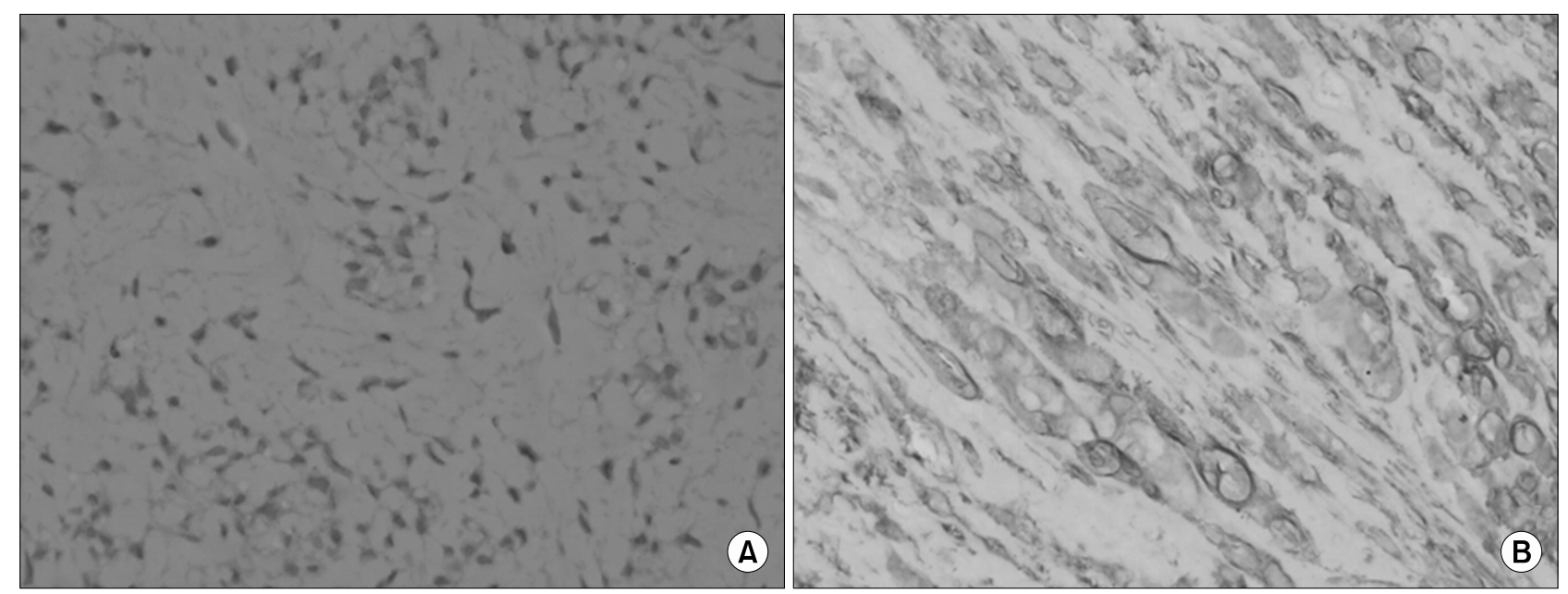

Fig. 4. The immunohistochemistry features were weakly positive for CD-117 (c-kit) (A) and smooth muscle actin expression (B).

tinues to do well.

\section{DISCUSSION}

GISTs are the most common mesenchymal tumors of the gastrointestinal track (GIT). Approximately $70 \%$ occur in the stomach, $20 \% \sim 30 \%$ in the small intestine, and less than $10 \%$ in the esophagus, colon, or rectum. Rare cases are identified outside the GIT and these are collectively known as EGISTs. ${ }^{3}$ The majority of EGISTs are found in the omentum, but it has also been identified in scrotum, bladder, pharynx, inguinal hernia, hypochondrium, pancreas, pelvis, retroperitoneum, and rectovaginal septum. ${ }^{4,5}$ In this case the tumor was originated from the crus muscle. Sometimes hepatic hemangioma, accessory spleen or pancreatic pseudocyst were mimicking subepitelial tumor of the stomach. Abdominal computed tomography scan showed that extrinsic compression of the stomach could be observed. But it was difficult to distinguish from exophytic tumor of esophagogastric junction area to extrinsic compression mass. Nearly all of those cases were clearly defined at the time of operation. If the tumor was originated from stomach wall the treatment was enough to wedge resection of stomach. But it was unrelated to the stomach we should carefully examined the origin of the tumor and clear removal was necessary. The clinicopathologic and histomorphologic features of GISTs and EGISTs are similar. Tumors may appear as well-defined or polylobulated firm masses with or without cysts, necrosis, or degeneration. They range in size from 2.1 to $32 \mathrm{~cm}$ and most are more than $5 \mathrm{~cm}$, because of the space available for growth, and are usually inseparable from the wall of the stomach or intestine, ${ }^{6}$ but the tumor of this patient was easily separated from the wall of stomach. Abdominal ultrasonography is the first-line screening modality used to detect the presence of a mass and to differentiate cystic and solid tumors. However, it does not usually enable identification of the primary site or tumor characteristics. CT and PET can identify the primary tumor site and the relationship between tumors and other organs. Based on immunohistochemical results, the EGISTs were positive for c-KIT in $96.4 \%$ (27/28) of samples, PKC- $\theta$ in $82.1 \%$ (23/28), DOG-1 in $85.7 \%$ (24/28), PDGFRA in $82.1 \%(23 / 28)$, CD34 in $67.9 \%$ (19/28), vimentin in $100 \%$ (28/28), $\alpha$-SMA in $28.6 \%(8 / 28), \mathrm{S}-100$ protein in $39.3 \%(11 / 28)$ and desmin in $28.6 \%(8 / 28)$. c-KIT, DOG-1 and PKC- $\theta$ immunostains were sensitive and specific, but the PDGFRA stain was not specific for EGISTs. ${ }^{7}$ In fact, EGISTs account for $7 \%$ of CD117 positive staining abdominal mesenchymal tumors. ${ }^{8}$ In our case, the tumor was weakly positive for CD-117 (c-kit) and smooth muscle actin expression. GISTs occur in the gastrointestinal tract and their prognosis are importantly influence by tumor size and mitotic count. On the other hand, for EGISTs, a mitotic count of greater than 5 in 50 high-power field of view, if cell necrosis within the tumor tissue or cells from the high-fidelity indicates poor prognosis. But tumor size was not related to the prognosis. ${ }^{9,10}$ Site, histologic type and omental origin mass is more poor prognosis than mesentery, even in the event of a multiple omental origin mass are more poor prognosis than occur in the single. ${ }^{3,10}$ In this case the tumor was single and the pathologic report was moderate risk gastrointestinal stromal tumor with less than 5/50 HPF, spindle cell type, no tumor necrosis, no tumor hemorrhage and smooth muscle differentiation. EGISTs are treated in the same manner as GISTs, that is, by complete primary tumor resection regardless of size. Prognoses 
in cases of complete resection are relatively good. ${ }^{10}$ In our case, there was no tumor necrosis, the mitotic count was 5 or less, and there was a single mass of more than $5 \mathrm{~cm}$. Nevertheless, the tumor was completely removed without leaving any lesion, and thus, prognosis is expected to be good.

In this case gastroscopic examination showed bulging mucosa, about $4 \times 3 \mathrm{~cm}$ in diameter in the posterior wall of the cardia. It was suspected subepitelial tumor and computed tomography (CT) depicted a well-defined exophytic tumor. So laparoscopic surgery was done. Operative findings indicated that the mass was independent of the stomach and originated from the right crus muscle. In cases of a peritoneal tumor of unknown origin, in various forms of other parts of the gastrointestinal tract can cause gastrointestinal stromal tumors, taking into account that they check for an active organization must be considered in attempts.

\section{REFERENCES}

1) Tateishi U, Hasegawa T, Satake M, Moriyama N. Gastrointestinal stromal tumor: correlation of computed tomography findings with tumor grade and mortality. J Comput Assist Tomogr 2003;27:792-798.

2) Yamamoto $H$, Oda $Y$, Kawaguchi $K$, et al. c-Kit and PDGFRA mutations in extragastrointestinal stromal tumor (gastrointestinal stromal tumor of the soft tissue) Am J Surg Pathol 2004;28:479-488.

3) Miettinen M, Monihan JM, Sarlomo-Rikala M, Kovatich AJ, Carr NJ, Emory TS. Gastrointestinal stromal tumors/smooth muscle tumors (GISTs) primary in the omentum and mesentery: clinicopathologic and immunohistochemical study of 26 cases. Am J Surg Pathol 1999;23:1109-1118.

4) Lam MM, Corless CL, Goldblum JR, Heinrich MC, DownsKelly E, Rubin BP. Extragastrointestinal stromal tumors presenting as vulvovaginal/ rectovaginal septal masses: a diagnostic pitfall. Int J Gynecol Pathol 2006;25:288-292.

5) Foster R, Solano S, Mahoney J, Fuller A, Oliva E, Seiden MV. Reclassification of a tubal leiomyosarcoma as an eGIST by molecular evaluation of c-KIT. Gynecol Oncol 2006;101: 363-366.

6) Matteo D, Dandolu V, Lembert L, Thomas RM, Chatwani AJ. Unusually large extraintestinal GIST presenting as an abdomino-pelvic tumor. Arch Gynecol Obstet 2008;278:89-92.

7) $\mathrm{Kim} \mathrm{KH}$, Nelson SD, Kim DH, et al. Diagnostic relevance of overexpressions of PKC- $\theta$ and DOG-1 and KIT/PDGFRA gene mutations in extragastrointestinal stromal tumors: a Korean six-centers study of 28 cases. Anticancer Res 2012; 32:923-937.

8) Lanzafame S, Minutolo V, Caltabiano R, et al. About a case of GIST occurring during pregnancy with immunohistochemical expression of epidermal growth factor receptor and progesterone receptor. Pathol Res and Pract 2006;202:119-123.

9) Reith JD, Goldblum JR, Lyles RH, Weiss SW. Extragastrointestinal (soft tissue) stromal tumors: an analysis of 48 cases with emphasis on histologic predictors of outcome. Mod Pathol 2000;13:577-585.

10) Miettinen M, Sobin LH, Lasota J. Gastrointestinal stromal tumors presenting as omental masses-a clinicopathologic analysis of 95 cases. Am J Surg Pathol 2009;33:1267-1275. 